\title{
An Operator Absorbing Boundary Condition for the Absorption of Electromagnetic Waves in Dispersive Media
}

DOI:

10.1109/TAP.2018.2796386

\section{Document Version}

Accepted author manuscript

Link to publication record in Manchester Research Explorer

\section{Citation for published version (APA):}

Abdulkareem, B., Bérenger, J. P., Costen, F., Himeno, R., \& Yokota, H. (2018). An Operator Absorbing Boundary Condition for the Absorption of Electromagnetic Waves in Dispersive Media. IEEE Transactions on Antennas and Propagation . https://doi.org/10.1109/TAP.2018.2796386

\section{Published in:}

IEEE Transactions on Antennas and Propagation

\section{Citing this paper}

Please note that where the full-text provided on Manchester Research Explorer is the Author Accepted Manuscript or Proof version this may differ from the final Published version. If citing, it is advised that you check and use the publisher's definitive version.

\section{General rights}

Copyright and moral rights for the publications made accessible in the Research Explorer are retained by the authors and/or other copyright owners and it is a condition of accessing publications that users recognise and abide by the legal requirements associated with these rights.

\section{Takedown policy}

If you believe that this document breaches copyright please refer to the University of Manchester's Takedown Procedures [http://man.ac.uk/04Y6Bo] or contact uml.scholarlycommunications@manchester.ac.uk providing relevant details, so we can investigate your claim.

\section{OPEN ACCESS}




\section{An Operator Absorbing Boundary Condition for the Absorption of Electromagnetic Waves in Dispersive Media}

Buraq Abdulkareem, Student Member, IEEE, Jean-Pierre

Bérenger, Fellow IEEE, Fumie Costen, Senior

Member, IEEE, Ryutaro Himeno, Hideo Yokota

\begin{abstract}
An absorbing boundary condition is presented for the absorption of electromagnetic waves in dispersive media in problems where the frequency bandwidth of interest is limited. The condition relies on an operator designed to absorb the waves in the considered bandwidth. Numerical experiments demonstrate the effectiveness of the operator in Human Body media. Due to its simple implementation, the operator ABC may be an alternative to the PML in applications where the bandwidth does not exceed one or two decades of frequency.
\end{abstract}

Index Terms-Maxwell equations, FDTD, Absorbing Boundary Condition, PML, dispersive media.

\section{INTRODUCTION}

In FDTD or any other time domain methods, the treatment of an open space at outer boundaries of computational space filled with dispersive media has been a challenging question for many years. Such Absorbing Boundary Conditions (ABCs) as the Engquist-Majda $\mathrm{ABC}$ or the Higdon $\mathrm{ABC}$ were not able to be used. Nowadays, we can apply the PML ABC regardless the frequency spectrum of the problem, in particular when the spectrum spans over several decades of frequency. However there exist time domain problems where the band of frequency is narrow, especially in bioelectromagnetics where the bandwidth of interest only spans over a fraction of a decade. In such problems, one may think that an $\mathrm{ABC}$ simpler than the PML could be elaborated, because the problem to be solved does not require the wide band feature of the PML. This is the main motivation of the introduction of a new and simple $\mathrm{ABC}$ in this paper. In principal, PML has number of parameters, mainly layer thickness, profile of conductivity, and theoretical absorption, must be set prior to simulations. In complex media, such as Debye media, an extensive study is needed to define the optimised PML parameters.

The proposed $\mathrm{ABC}$ is an operator designed to absorb travelling waves in dispersive media. The operator is frequency dependent and thus it is only exact at a specified frequency. However it can provide with an acceptable approximation within a bandwidth larger than the bandwidth of interest of many problems of bioelectromagnetics. Moreover the practical bandwidth of the $\mathrm{ABC}$ can be enlarged by using the HABC principle [1] which allows several operators to be easily combined.

B. Abdulkareem, J.-P. Bérenger, F. Costen are with the School of Electrical and Electronic Engineering, The University of Manchester, U.K. (email: fumie.costen@manchester.ac.uk)

R. Himeno is with the Advanced Center for Computing and Communication, RIKEN, Saitama, Japan

H. Yokota and F. Costen are with the Image Processing Research Team, Center for Advanced Photonics, RIKEN, Saitama, Japan.

Additional research data supporting this publication are available from the http://dx.doi.org/ repository at 10.15127/1.306073.
Section $\Pi$ introduces the $\mathrm{ABC}$ and derives and discusses its reflection. It is shown that it is possible to effectively absorb waves in bandwidths as large as one or two decades in the Human-Body Debye media. In Section III] FDTD numerical simulations are reported in the two-dimensional case. One consists of absorbing the field radiated by a source placed in front of a plane operator $\mathrm{ABC}$. The other deals with the field scattered by a perfect electric conductor (PEC). Both simulations demonstrate the effectiveness of the $\mathrm{ABC}$.

\section{The OPERATOR ABC FOR Dispersive Media}

Let us consider a dispersive medium where the travelling waves are absorbed as they propagate. The wavenumber $k$ is then complex and can be written as

$$
k=\alpha-\jmath \beta
$$

where $\alpha$ and $\beta$ depend on frequency and are proportional to the real and imaginary parts of the complex permittivity $\epsilon_{r}$ of the medium. The $\alpha$ and $\beta$ are given by

$$
\alpha=\frac{\omega}{c} \operatorname{Re}\left(\sqrt{\epsilon_{r}}\right) \quad ; \quad \beta=-\frac{\omega}{c} \operatorname{Im}\left(\sqrt{\epsilon_{r}}\right)
$$

where $\omega$ is the angular frequency, and $c$ is the speed of light in vacuum. If the propagation is in $x$ direction, the waveform reads

$$
U=U_{0} e^{\jmath \omega(t-x / v)} e^{-\beta x}
$$

where $U$ is the magnitude of the wave, and $v=\omega / \alpha$ is the velocity of the phase. The derivatives on time and space of (2) are then

$$
\begin{gathered}
\frac{\partial U}{\partial t}=\jmath \omega U, \\
\frac{\partial U}{\partial x}=-\jmath \frac{\omega}{v} U-\beta U .
\end{gathered}
$$

Substitution of $(3)$ into $(4)$ yields

$$
\frac{\partial U}{\partial x}+\frac{1}{v} \frac{\partial U}{\partial t}+\beta U=0
$$

which is a time domain equation. Notice that when $\beta=0$ it reduces to the first order equation of Engquist-Majda [2] or Higdon [3]|4]. The term $\beta U$ in (5) takes into account the attenuation of the wave. Such a term was introduced in the past [5] to reduce the late time instability that may occur with the Higdon operators. It was also used to improve the absorption of evanescent waves [6][7]. Equation (5) is frequency dependent since both $v$ and $\beta$ depend on frequency in physical media, especially in lossy and Debye media. The proposed $\mathrm{ABC}$ consists of using (5) in direction $x$ perpendicular to the boundary and at a prescribed frequency of design $f_{0}$. When parameters $v_{0}$ and $\beta_{0}$ corresponds to $f_{0}$, (5) can be written as

$$
\frac{\partial U}{\partial x}+\frac{1}{v_{0}} \frac{\partial U}{\partial t}+\beta_{0} U=0 .
$$

In order to implement (6) as an $\mathrm{ABC}$ in such a finitedifference method as the FDTD method, we discretize (6) on a line perpendicular to the boundary, as shown in Fig. 1 Denoting $U_{0}$ as the field at the node on the boundary, $U_{-1}$ as 


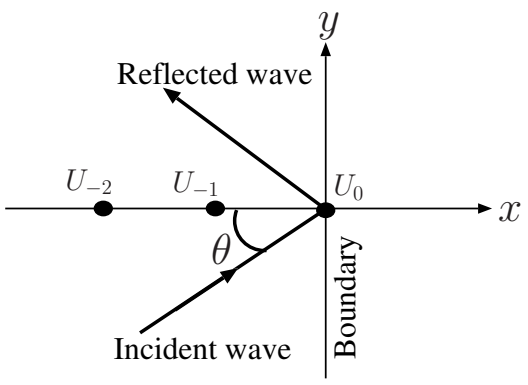

Fig. 1. An incident plane wave striking a plane boundary at incidence $\theta$

the field at the node one spatial step $\Delta x$ from the boundary, and considering FDTD time steps $n$ and $n+1$ separated with $\Delta t$, a discretization of (6) using the central difference approximation, similar to the one used in [3][4] [5], yields the unknown field $U_{0}$ at time $n+1$ in function of known $U_{0}$ at time $n$ and $U_{-1}$ at times $n$ and $n+1$ as

$$
U_{0}^{n+1}=W_{1} U_{-1}^{n}+W_{2} U_{-1}^{n+1}-W_{3} U_{0}^{n}
$$

where

$$
\begin{aligned}
W_{1}= & \left(v_{0} \Delta t+\Delta x-\beta_{0} \Delta x v_{0} \Delta t / 2\right) / D, \\
W_{2}= & \left(v_{0} \Delta t-\Delta x-\beta_{0} \Delta x v_{0} \Delta t / 2\right) / D, \\
W_{3}= & \left(v_{0} \Delta t-\Delta x+\beta_{0} \Delta x v_{0} \Delta t / 2\right) / D, \\
& D=v_{0} \Delta t+\Delta x+\beta_{0} \Delta x v_{0} \Delta t / 2 .
\end{aligned}
$$

Notice that (7) is in the same form as the discretization of the Higdon operator where $W_{1}=1$ and $W_{2}=W_{3}=(c \Delta t-$ $\Delta x) /(c \Delta t+\Delta x)$. Equation (7) is exact at normal incidence and at frequency $f_{0}$. At any other incidence or frequency, the $\mathrm{ABC}$ in (7) will produce some reflection. Derivation of the theoretical reflection can be performed as with previous operator $\mathrm{ABCs}$ [4][5][7]. Let us consider a plane wave of frequency $f$ propagating in angle $\theta$ as depicted in Fig. 1. The incident and reflected waves can be written as

$$
\begin{aligned}
E_{i}(x, y, t) & =E_{0 i} e^{\jmath \omega t-\jmath k_{x} x-\jmath k_{y} y} \\
E_{r}(x, y, t) & =E_{0 r} e^{\jmath \omega t+\jmath k_{x} x-\jmath k_{y} y}
\end{aligned}
$$

where $k_{x}=k \cos \theta$ and $k_{y}=k \sin \theta$ are the components of wavenumber $k$ in (1). The sum of the two waves must satisfy (7) on the boundary. Substituting (97) into (7) the reflection $r=E_{0 r} / E_{0 i}$ is obtained as

$r=-\frac{1-W_{1} e^{-\jmath \omega \Delta t} e^{\jmath k_{x} \Delta x}-W_{2} e^{\jmath k_{x} \Delta x}+W_{3} e^{-\jmath \omega \Delta t}}{1-W_{1} e^{-\jmath \omega \Delta t} e^{-\jmath k_{x} \Delta x}-W_{2} e^{-\jmath k_{x} \Delta x}+W_{3} e^{-\jmath \omega \Delta t}}$

Reflection (10) depends on the parameters $v_{0}$ and $\beta_{0}$ through $W_{1}, W_{2}, W_{3}$, and on $v$ and $\beta$ through $k$.

As with other operators, a close form of the reflection can be derived assuming that the frequency is far from the cutoff frequency of the numerical method, which in lossy media reads $\Delta t \ll 1 / \omega, \Delta x \ll \lambda$ and $\Delta x \ll \delta$, where $\lambda=v / f$ is the wavelength and $\delta=1 / \beta$ is the skin depth. With such assumptions, (10) reduces to

$$
r=\frac{v v_{0}\left(\beta_{0}-\beta \cos \theta\right)+\jmath \omega\left(v-v_{0} \cos \theta\right)}{v v_{0}\left(\beta_{0}+\beta \cos \theta\right)+\jmath \omega\left(v+v_{0} \cos \theta\right)} .
$$

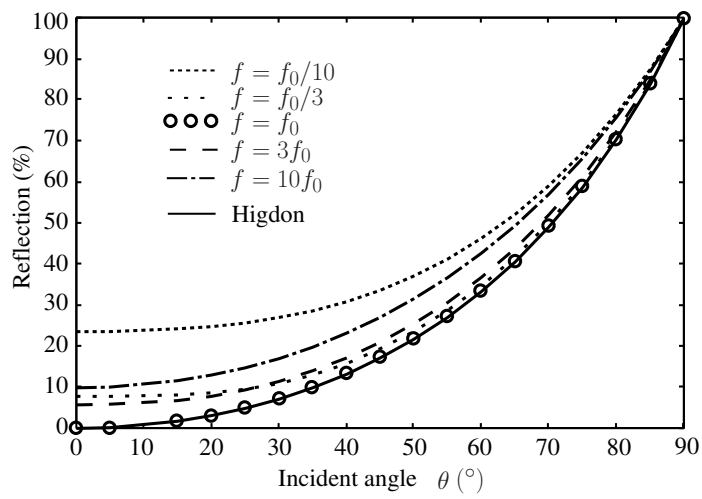

Fig. 2. Reflection (11) in Skin medium in function of the incidence angle at the design frequency $f_{0}$ and at $f_{0} / 10, f_{0} / 3,3 f_{0}, 10 f_{0}$. The Higdon reflection is that of the first order operator in vacuum.

At the frequency of design of the operator, we have $v=v_{0}$ and $\beta=\beta_{0}$, and thus (11) reduces to

$$
r=\frac{1-\cos \theta}{1+\cos \theta}
$$

which is nothing but the reflection produced by the Higdon operator in a vacuum.

Reflection (11) in Skin medium is plotted in Fig. 2 as a function of $\theta$ at frequencies $f_{0} / 10, f_{0} / 3, f_{0}, 3 f_{0}, 10 f_{0}$ where $f_{0}=1 \mathrm{GHz}$. Skin tissue is modelled as a first order Debye medium whose relative permittivity is

$$
\epsilon_{r}(\omega)=\epsilon_{\infty}+\frac{\epsilon_{\mathrm{S}}-\epsilon_{\infty}}{1+\jmath \omega \tau_{D}}+\frac{\sigma}{\jmath \epsilon_{0} \omega}
$$

where $\epsilon_{0}$ is the free space permittivity, the static relative permittivity $\epsilon_{\mathrm{S}}=47.9$, the optical relative permittivity $\epsilon_{\infty}=$ 29.9 , the conductivity $\sigma=0.54 \mathrm{~S} / \mathrm{m}$ and the relaxation time $\tau_{D}=43.6$ ps. As expected the reflection at $f=f_{0}$ is superimposed to the Higdon operator reflection in (12). As frequency deviates from $f_{0}, r$ in (11) increases most at about normal incidence, where it is about $23 \%$ at $f_{0} / 10$.

Thus, the expected effectiveness of the proposed $\mathrm{ABC}$ can be characterised by the reflection at normal incidence, where the difference between $r$ in (11) and $r$ in 12 is maximum. Reflection $r$ in (11) at $\theta=0$ is plotted in Fig. 3 for four media. The behaviors of the reflection in function of frequency are similar. However, Fig. 3 shows that the more dispersive the medium is the higher the reflection.

Operator ABCs can be combined by multiplying several first order operators in the sense of Higdon [4][5]. The resulting reflection is then the product of the elementary reflections. An equivalent and simpler method to obtain high order reflection consists of implementing the operators by the Huygens absorbing boundary condition (HABC) method [1][10]. Then, a high order reflection can be obtained by simply juxtaposing several first order operators in series in the FDTD grid [1]. Multiplication of operators provides the possibility of extending the frequency band where the reflection is low at normal incidence, and making the resultant reflection close to the Higdon reflection. This can be realised by using several operators in (7), each of which works at different frequency 


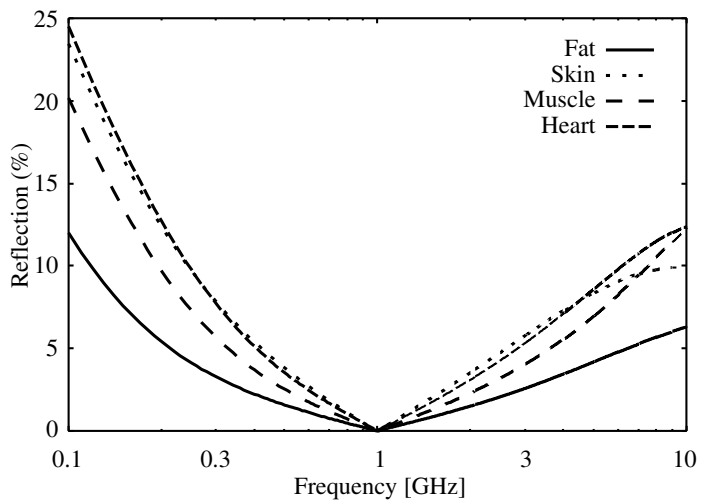

Fig. 3. Reflection (11) at normal incidence in a 2-decade bandwidth about the design frequency $f_{0}$. The parameters $\left(\epsilon_{\mathrm{S}}, \epsilon_{\infty}, \sigma, \tau_{D}\right)$ of Fat, Muscle, Heart are (5.5, 3.998, 0.037 S/m, $23.6 \mathrm{ps}),(56.9,28,0.75 \mathrm{~S} / \mathrm{m}, 18.7 \mathrm{ps})$, $(63.6,34.9,1.02 \mathrm{~S} / \mathrm{m}, 28.9 \mathrm{ps})$ respectively. These parameters are obtained by fitting the one-pole Debye media parameters of human tissues [8] using the measurement data in [9].

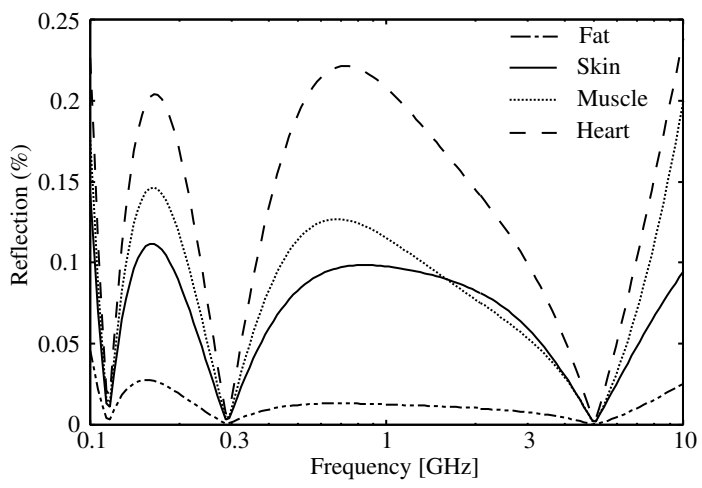

Fig. 4. Reflection from the 3-order operator at normal incidence with design frequencies optimised for Skin within a 2-decade bandwidth. The design frequencies are $0.115 \mathrm{GHz}, 0.29 \mathrm{GHz}$, and $5 \mathrm{GHz}$.

of design. This is demonstrated in Fig. 4 and Fig. 5 where the $\mathrm{ABC}$ is composed of three operators in (7).

In Fig. 4, the design frequencies of the operators were optimised to make the reflection in the Skin medium as low as possible in a bandwidth of two decades, from 0.1 $\mathrm{GHz}$ to $10 \mathrm{GHz}$. Comparing with the first order operator in Fig. 3, the improvement is large where the reflection does not exceed $0.25 \%$ in the whole 2-decade bandwidth. When the desired bandwidth is only one decade, three operators allow the reflection to be lower than $0.01 \%$ as shown in Fig. 5. This is negligible in most numerical calculations, where there are other sources of noise.

In summary, the reflections plotted in Fig. 4 and Fig. 5 show that cascading several operators makes the reflection similar to that produced by the Higdon operator in vacuum in a significant bandwidth of one or two decades. The reflection is negligible about normal incidence, and remains low up to wide angles because three operators make the reflection the cube of (12) at wide angles. The operator (7) may be effective in many applications of numerical methods where the actual bandwidth of interest is by far narrower than one or two decades.

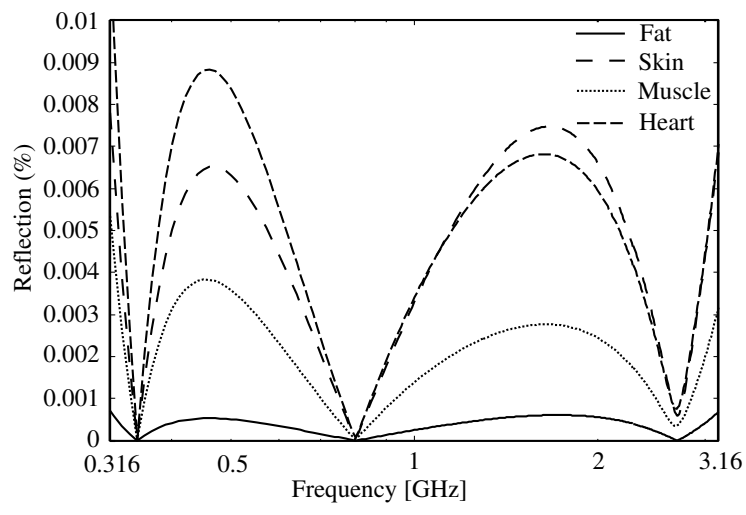

Fig. 5. Reflection from the 3-order operator at normal incidence with design frequencies optimised for Skin within a 1-decade bandwidth. The design frequencies are $0.35 \mathrm{GHz}, 0.8 \mathrm{GHz}$, and $2.7 \mathrm{GHz}$.

\section{NUMERICAL EXPERIMENTS}

The capability of the proposed operator $\mathrm{ABC}$ to absorb signals from the source excitation or reflected by a PEC object is examined numerically in the two-dimensional FDTD space which is filled with Human tissue of Fat in Section III-A and Section III-B The one-pole Debye parameters for Fat are $\epsilon_{\mathrm{S}}=5.53, \epsilon_{\infty}=3.998, \sigma=0.037 \mathrm{~S} / \mathrm{m}, \tau_{\mathrm{D}}=23.6 \mathrm{ps}$. The excitation waveform is

$$
H_{z}(t)=100 / 377 \quad \exp ^{-\left(\frac{t-3 \tau}{\tau}\right)^{2}} \sin \left(2 \pi f_{m} t\right)
$$

where $\tau=1$ ns and $f_{m}=1 \mathrm{GHz}$. The normalised spectrum $\mathcal{S}(f)$ of (14) satisfies $0.1 \leq|\mathcal{S}(f)| \leq 1$ for $0.5 \mathrm{GHz} \leq f \leq 1.5$ GHz. The operators are implemented by the HABC method which permits the 3-order operator to be replaced with three 1order operators separated with two FDTD cells. The distance between the outermost HABC and the PEC boundary is 3 FDTD cells. The design frequency of the 1-order operator is $1 \mathrm{GHz}$ whilst the 3-order operator is the 1-decade operator in Fig. 5

\section{A. Absorption of travelling waves from the source excitation}

A hard source [11] is impressed at an $H_{z}$ node, $d$ cells away from an $\mathrm{ABC}$ plane where $d$ is set to 10 or 50 as depicted in Fig. 6 Component $H_{z}$ is observed $2 \Delta x$ away from the HABC when the spurious reflections from the other three sides of the FDTD space are not visible by using a large enough FDTD space. A free space solution was obtained without HABC within an enlarged FDTD space as shown in Fig. 6.

Fig. 7 shows the observations for the two cases of $d=10$ and $d=50$. The free space solution and a solution computed with the PML ABC are plotted for comparison. As expected, the result with the 3-order operator matches the free space better than that of the 1-order operator. When the distance $d$ between the source and the HABC increases the wavefront which strikes the HABC becomes closer to a plane wave at normal incidence and the match improves. However, even when the source is only 10 cells away from the HABC, the match is satisfactory with the 3-order operator which widens 


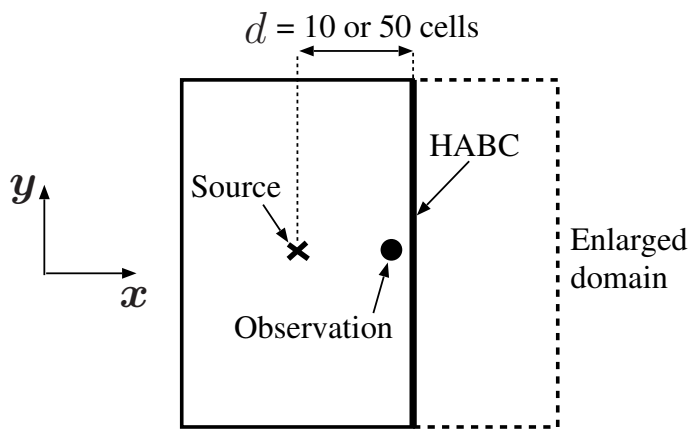

Fig. 6. FDTD space for the absorption of a spherical wave by a plane HABC and for the calculation of a free space solution. The steps are $\Delta x=\Delta y=0.5$ $\mathrm{mm}$ and $\Delta t=1 \mathrm{ps}$. The three components of the 2D field are $E_{x}, E_{y}$, and $H_{z}$. The source is enforced at an $H_{z}$ node and $H_{z}$ is observed two cells from the HABC. The whole space is filled with Fat medium.

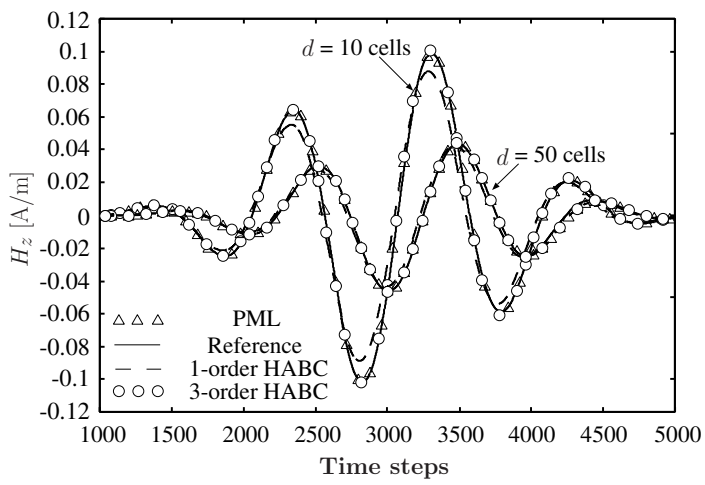

Fig. 7. $H_{z}$ observed in the scenario of Fig. 6 to examine absorption of an outgoing wave by 1 -order and 3-order operators in Fat medium. The source is placed either 10 or 50 FDTD cells from the operator.

the design bandwidth of the operator and reduces the wideangle reflection. Note that a PML with thickness of 10 cells achieves a similar accuracy to the 3 -order operator HABC with larger computational cost.

\section{B. Absorption of signals scattered by a PEC object}

An incident plane wave generated by the Huygens surface method [12] strikes a PEC object placed in Fat medium as depicted in Fig. 8 The $\boldsymbol{E}$ field perpendicular to the object surface at $0.5 \Delta y$ away from the object is observed at the end of the object. Fig. 9 shows the observation together with the free space solution obtained without HABC within the FDTD space of $2000 \times 2000$ cells and a solution for the case when the HABC in Fig. 8 is replaced with a PEC boundary. Despite the short distance of 4 cells between the object and the HABC, the 1-order solution is close to the free space solution and the 3 -order solution is almost perfectly superimposed on the free space solution.

\section{Other media and other frequency ranges of interest}

We have performed experiments with other human body media. For instance the observed results with Skin, which are not reported here, match the free space solution better than the ones in the case of Fat shown in Fig. 7 and Fig. 9 This

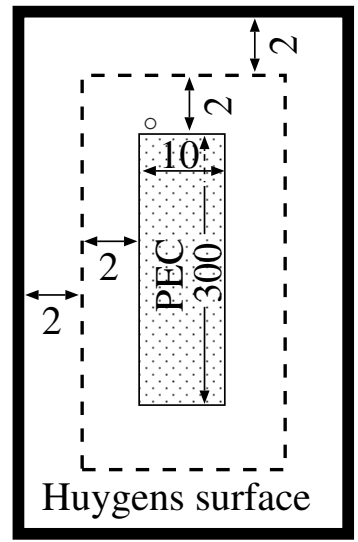

$\mathrm{HABC}$

Fig. 8. FDTD space for absorption of the field scattered by a PEC object. The FDTD steps are $\Delta x=\Delta y=1 \mathrm{~mm}$ and $\Delta t=2 \mathrm{ps}$. The space outside the PEC object is filled with Fat medium. Symbol $\circ$ shows the location of the observation.

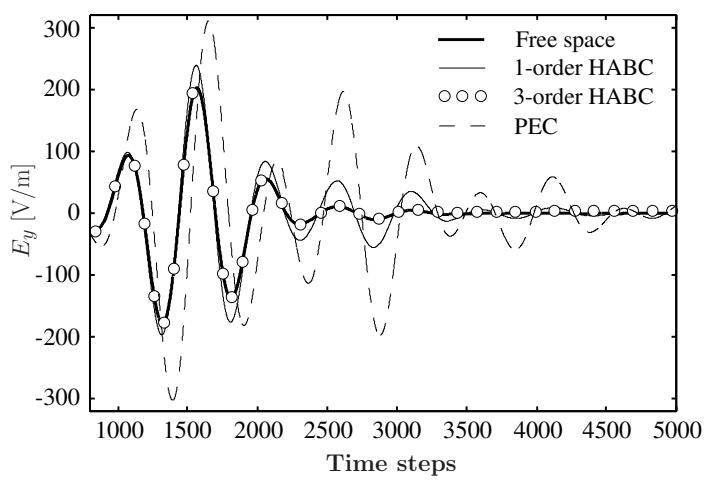

Fig. 9. $E$ field normal to the surface of the PEC object computed with the 1 -order and 3-order operators placed 4 cells from the object.

is because the absorption in Skin is higher than that in Fat and thus the observed field in Skin is less than that in Fat notwithstanding that the theoretical reflection of the proposed $\mathrm{ABC}$ is higher in Skin. In general, the higher the absorption in the medium is, the lower the residual reflection from the $\mathrm{ABC}$.

We also performed numerical simulations with an unmodulated Gaussian pulse as excitation waveform. A spurious reflection at the low frequencies was observed as expected. This confirms that the operator is effective when the bandwidth of the problem is within the design bandwidth of the operator.

\section{CONCLUSION}

An operator $\mathrm{ABC}$ has been elaborated for the absorption of travelling waves in dispersive media when the spectrum of the problem is limited to a band of frequencies. The $\mathrm{ABC}$ is effective with Human Body Debye media, as demonstrated by simulations. It can be used as well with other dispersive media. The implementation in the FDTD method is simple, especially using the HABC technique [1][10] that permits easy combination of operators to achieve a high-order $\mathrm{ABC}$ and to enlarge the bandwidth that is covered by the ABC. Thus 
the operator $\mathrm{ABC}$ appears as an interesting alternative to the PML ABC in some applications where the frequency band of interest does not exceed one or two decades, in particular in bio-electromagnetism calculations. A weakness of the $\mathrm{ABC}$ may be the absorption of evanescent waves which is a critical question with operator ABCs in vacuum [7]. This could be the matter of further investigations on the combination of the operator (6)-(7) designed for travelling waves with operators designed for evanescent waves, in the manner discussed in [7].

\section{ACKNOWLEDGEMENT}

This work was completed in part with HOKUSAIGreatWave Computer System provided by the RIKEN Advanced Center for Computing and Communication www.riken.jp/en/research/labs/accc.

\section{REFERENCES}

[1] J.-P Bérenger. On the Huygens Absorbing Boundary Conditions for Electromagnetics. J. Comput. Phys., 226, 354-378, Apr 2007.

[2] B. Engquist and A. Majda. Radiation Boundary Condition for The Numerical Simulation of Waves. Math. Comput., vol. 31, pp. 629-651, 1977.

[3] R. Higdon. Absorbing boundary conditions for difference approximations to the multi-dimentional wave equation. Math. of Comput., 47:437459, 1986.

[4] R. Higdon. Numerical Absorbing Boundary Conditions for The Wave Equation. Math. of Comput., 49:65-90, 1987.

[5] R. L. Higdon. Radiation boundary conditions for elastics wave propagation. SIAM J. Numerical Analysis, 27:831-870, 1990.

[6] V. Betz and R. Mittra. Comparison and Evaluation of Boundary Conditions for Absorption of Waves in an FDTD Simulation. IEEE Micr. Guid. Waves Lett., vol. 2, pp. 499-501, 1992.

[7] J.-P. Bérenger. A Histroical Review of The Absorbing Boundary Conditions. FERMAT, vol. 9, 2015.

[8] T. Wuren, T. Takai, M. Fujii, and I. Sakagami. Effective 2-Debye-Pole FDTD Model of Electromagnetic Interaction Between Whole Human Body and UWB Radiation. IEEE Microwave Wireless Comp. Lett., 17(7):483-485, 2007.

[9] S. Gabriel, R. W. Lau, and C Gabriel. The Dielectric Properties of Biological Tissues: III. Parametric Models for the Dielectric Spectrum of Tissues. Phys. Med. Biol., 41:2271-2293, 1996.

[10] F. Costen and J.-P Bérenger. Implementation of The Huygens Absorbing Boundary Condition in Corner Regions. IEEE Trans. Electrom. Compat., 54, pp. 367-374, 2012.

[11] F. Costen, J.-P. Bérenger, and A. K. Brown. Comparison of FDTD Hard Source With FDTD Soft Source and Accuracy Assessment in Debye Media. IEEE Trans. Antenna and Propag., 57(7), July 2009.

[12] B. Abdulkareem, J.-P Bérenger, and F. Costen. Huygens Excitation in Debye Media in The FDTD. IEEE Trans. Antennas Propag., vol. 64, no. 8, pp. 3632-3635, 2016. 